\title{
A FEDERATED LEARNING SYSTEM FOR OPTIMISED ENVIRONMENTAL CONTROL OF CONSECUTIVE AREAS
}

\author{
Robert Perry and Enda Fallon \\ Software Research Institute, Athlone Institute of Technology, Athlone, Ireland
}

\begin{abstract}
Building management systems have led to artificially controlled environmental conditions. While new infrastructure will lead to a lower carbon footprint and a better working environment, the costs cannot justify the rewards at this present time. Ambient temperature regulation has the potential to mitigate excessive energy consumption. This work proposes an externally influenced environmental control Artificial Neural Network (ANN) implementation to optimise ambient temperature for a given specific area whether that be internal or external to the building. The approach uses a multi-aspect ANN. Two architectural components are introduced, an Agent ANN (A-ANN) and a Coordinating ANN (C-ANN). The Agent ANNs (A-ANN) are deployed to provide temperature control at the extremities of the open plan area. The A-ANN operates with a degree of autonomy. A Coordinating ANN (C-ANN) considers the optimal ambient temperature of the room and consequently effects the surrounding area(s). These A-ANNs have internal and external factors acting as effectors to the system such as outdoor environmental conditions and internally located effectors such as adjacent rooms. Results are presented which diagnose the effort applied by A-ANN instances in varying environmental conditions both internally and externally.
\end{abstract}

\section{KEYWORDS}

Artificial Neural Networks, Ambient Temperature Control, Building Management Systems

\section{INTRODUCTION}

Heating Ventilation and Control (HVAC) systems are contributing to the ongoing damage we inflict on our planet. In the United States of America, one of the highest pollution factors is from the industrial and commercial electricity sector of which HVAC units play a major part (Ueda, \& Shimonaka, 2017; Edenhofer, et al, 2014). In many environments these systems are left on for extended periods of time or are increased to temperatures which make the room uncomfortable and at times unusable. Open plan areas are a common architectural construct. Recent adoption of work practices such as the "Agile Way of Working" in the software design sector has coupled the design of a building to the working methodology. Agile software methodologies introduce the concept of "Informative Workspaces". The tightly coupled relationship between building and methodology has resulted in the reconfiguration of older buildings towards physical open plan seating. The reorganisation of such spaces can lead to an imbalance in temperature profile, tenant discomfort and excessive running costs through higher gas and electricity consumption. Buildings are often reconfigured without consideration of air flow, solar gain or the revised meaning of temperature sensor readings.

This work proposes a federated ANN configuration with two new architectural components an Agent ANN (A-ANN) and a Coordinating ANN (C-ANN). The A-ANN operates with a degree of autonomy with some federated influence from the C-ANN. The C-ANN considers the optimal ambient temperature of the entire open plan area. Having previously created a theoretical model and simulated results (Perry, Fallon, Fallon, \& Qiao, 2017) this work proposes an experimentally based evaluation of the architecture using a physical scaled model. The model enables the analysis of dispersion of heat between rooms in an office environment consisting of meeting rooms and a central open plan area. The work illustrates how a federated ANN approach consisting of both centralised and distributed intelligence can learn the optimal of HVAC actuators so as to optimise thermal flow throughout the office space.

This paper is organised as follows. Related work is discussed in section 2. An overview of ANN architectures is provided in Section 3. The system architecture of our approach is presented in Section 4. An experimental system evaluation is provided in Section 5. Finally, conclusions are described in Section 6. 


\section{RELATED WORK}

In (Matta, Rehu, Tanner, \& Kansala, 2017) the authors attempt to show the importance for both the control and monitoring of HVAC units with regard to the health and well-being of employees. Monitoring air quality is becoming an ever-increasing worry for most countries with many simply turning a blind eye to the damage that is happening with little action. Using a relatively new technology Bluetooth low energy, a range of monitoring devices were deployed as part of this research to ascertain the range of human effectors which are sometimes unconsciously present in an everyday commercial office building. Using temperature, humidity and air pressure metric sensing the research outlines a range of practically implemented points in a building that were monitored and the location of the HVAC unit associated with them. Updated on a minute by minute basis the research gains an accurate picture of what the environment most of us breath in and out in an office environment. Although this research only proposes future work in regard to the actual control of the environment the results show how the temperature and humidity variance in an office greatly increases and decreases dependant on the area being monitored at a given time. This research in similar in the way that monitoring the given environment is essential to gain an accurate picture of what this research plans to show. Air quality optimisation and HVAC control are problems that most advanced economies deal with on a daily basis and yet in most cases choose to ignore. It is the proposal of this research to find solutions to this extremely high drain on our energy consumption and show how we might increase the quality of the air that we breath concurrently, find other work which proposes to do the same and build upon existing infrastructure to try to solve inefficiencies.

In (Hsiung, \& Lin, 2018) the authors present a cyber-physical system with user-optimized control strategy that trades off between power consumption and user-specific comfort for building energy management. The work differs from our approach as the consideration is the optimisation of power consumption rather than ambient temperature regulation. In adopting such an approach, the authors show how a predicated mean voting mechanism can be substituted in the absence of a set of weights where a seven-point thermal scale is used to predict the comfort levels of a particular set of individuals. The flaw that this research presents however is in the consideration of the thermal comfort of a particular subject and not the efficiency of adjacent areas combined with the assumption that the research data collected will be absent of human error. At a contrast the research outlined here will show how historical data will be collected on an ongoing basis to account for thermal comfort but also take into consideration the environmental factors that exist as a result.

Furthermore to (Hsiung, \& Lin, 2018), the authors in (Ardiyanto, Pipattanasomporn, Rahman, Hariyanto, $\&$ Suwarno, 2018) express how thermal comfort objectives are not being met in buildings and how employees do not feel they are adequately comfortable, hence a predictive mean system could be introduced to allocate the setpoint temperatures more efficiently throughout a building. the authors devise a practical solution powered by a raspberry pi using Radio frequency identification to detect the occupancy and hence allocate the setpoints throughout the building to different HVAC area controllers. Using the eQuest simulation tool the authors implement show the building plan of a system located in Alexandria showing an efficiency increase of $14.58 \%$ when compared with a system not using the mean voting system. The research in addition outlines additional criteria for which an additional $8.79 \%$ may also be met given times of occupancy being accounted for. In (Gopika, 2015) a more holistic approach to the measurement of metrics is attained with the discovery of the thermal and energy inefficiency of a building using real-time data logging from multiple sources adding a more historical element to that of (Hsiung, \& Lin, 2018) or (Ardiyanto, et al, 2018). This approach shares convention with some existing smart controller HVAC systems and does not take the subjects comfort level as an input metric however this research does show how the application of neural network algorithm can improve performance and adaptability when studied from afar and excluding human decision factors and consequently human error.

Given the many advancements we have seen as part of artificial intelligence design in the software industry in recent years. The focus of this research should be to remove as much human control as possible and to let an algorithm decide based on historical data the optimal conditions that humans can thrive in based in minimal human influence negating as much human error as possible. In studying this research (Gopika, 2015), the resounding overall theme is similarly present notwithstanding the contrast being this paper proposes that we use the underlying infrastructure which may already be present in our building(s) in a more intelligent way while also factoring in human input and not discounting it completely. (Vatanparvar, Burago, \& Abdullah, 2018) Accurately describes how a historical collation of user behaviour in conjunction with a neural network 
and user input absorption over an extended time period are used to gain a predictive behavioural analysis over driving characteristics that one may accomplish greater power efficiency in an electric vehicle. Similarly planned to be shown in this research metrics are collected and allocated weights given a pre-determined set of factors. This research shows how in the automotive industry similar research exists but is at an early stage ready to be improved upon, this is similar to the HVAC industry however in contrast that for a long time period without the need to increase battery saving techniques, the problem was left to exist, this was interesting in the way that it poses a question many devices outside of the automotive or HVAC industries today could probably be coded more efficiently if there was a need to reduce the energy consumed when not directly or indirectly connected to the power grid.

Finally, the work in (Kim, 2018) focuses on a similar challenge HVAC Systems in Multizone Office Buildings Considering Thermal Preferences of Individual Occupants. The work shows how occupants thermal preference, a neural network modelling algorithm, a grid tie strategy and variable speed heat pumps are used to enhance productiveness and gain a reduction in energy consumption, similar to this approach the research outlined plans to show the practical application of how monitoring information, a neural network, historical data and a reduced element of user input metrics might be used in a neural network to reduce power network drain and accurately pinpoint spikes in demand throughout the day at a given area hence reducing the cost to maintain existing infrastructure and reform transmission loses proportionately.

\section{ARTIFICIAL NEURAL NETWORKS}

ANN are data processing models which are based on the operation of the brain. The first work on ANN was presented by McCullock and Pitts in 1943. The work proposed a Threshold Logic Unit (TLU) which used weighted binary inputs. If the weighted sum of inputs exceeded a threshold value, the neuron fired. Many enhancements to the original model have been introduced. The first unsupervised learning approach, Hebbian Learning, was proposed in 1949. Classification of inputs was introduced by the perceptron model. The introduction of back propagation enabled the training of synaptic weights based on a desired output. Modern ANN consist of a large number of interconnected neurons working in parallel, typically used for pattern recognition and data classification.

Figure 1 illustrates a supervised learning ANN. Values $X_{0}, X_{1}, X_{2} \ldots . X_{n}$ are provided as input to the neuron. The neuron has 2 modes of operation; training or trained. In trained mode, the neuron applies synaptic weights wk0, wk1....wkn which enhance or degrade the input values. These weighted values are summed and an activation function $\varphi($.$) is applied. \varphi($.$) determines whether the neuron should "fire", producing an output yk$ which classifies the input pattern. Training mode can be implemented through supervised or unsupervised learning. In supervised learning the ANN will have an offline training phase in which neural outputs are compared against a training set. Alterations are made to the synaptic weights to limit the error in classification between the output yk and the training set $\mathrm{dk}$. When the ANN correctly classifies the input pattern, the ANN operates in trained mode. Unsupervised learning has no external training patterns. In this mode the ANN self organizes data presented to the network and detects recurrent properties.

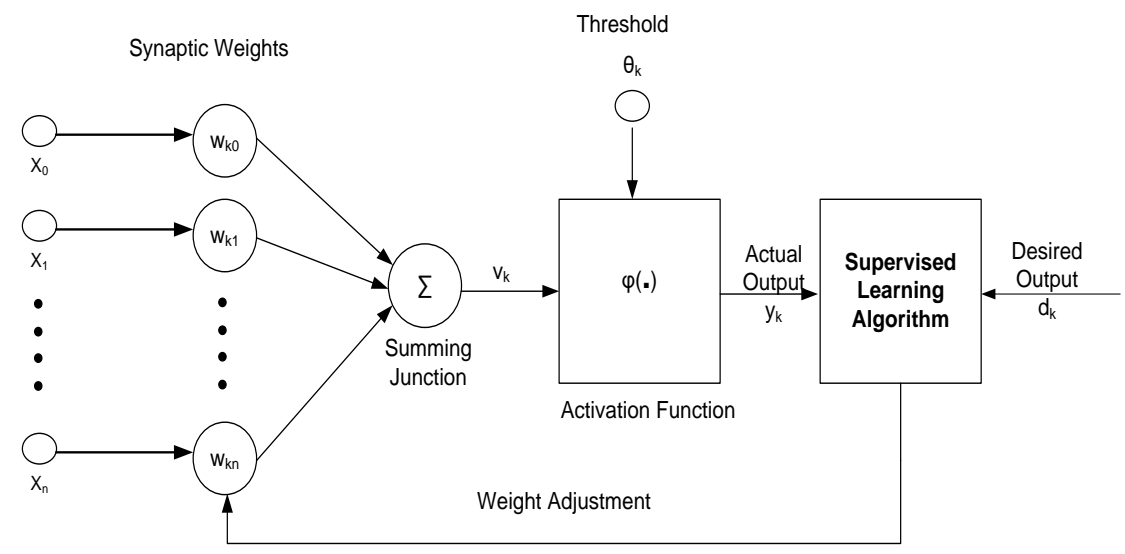

Figure 1. A Supervised Learning Neural Network 


\section{SYSTEM ARCHITECTURE}

This section describes the architecture of our federated artificial neural network, building on research undertaken in Perry, Fallon, Fallon, and Qiao, 2017. We illustrate how the system may be implemented to practically monitor the metrics and control the actuation of heating the ventilation controls. The approach uses a multi-phase ANN. Two architectural components are introduced an Agent ANN (A-ANN) and a Coordinating ANN (C-ANN). The A-ANN operates with a degree of autonomy. The C-ANN considers the optimal ambient temperature of the entire open plan area and influences the decisions of individual A-ANN in order to achieve a collectively balanced temperature. Figure 2 represents multiple implementations of Figure 1 in an exploded format. Each declaration of $R_{n}$ is a full implementation a learning network. The approach consists of $\mathrm{X}_{0}, \mathrm{X}_{1}, \ldots \mathrm{X}_{\mathrm{n}}$ neuron inputs corresponding to the performance metrics; outdoor temperature, Open Plan Area Temperature and Meeting Room 1 temperature. $\mathrm{X}_{0} \mathrm{R}_{0}$ corresponds to the current value of indoor temperature for meeting room $\mathrm{R}_{0} . \mathrm{W}_{0} \mathrm{R}_{0}$ represents the synaptic weight applied to the current value of indoor temperature for meeting room $\mathrm{R}_{0}$. Figure 2 illustrates the combined configuration of theses inputs with respect to their neural network allocated area. A-ANN neurons representing 3 meeting rooms affecting the temperature of the overall open plan area. While each A-ANN has a degree of functional autonomy each is coordinated by the C-ANN through the ancillary weight assessment. The C-ANN coordinates the ambient temperature for the main area within the open plan represented in Figure 2 as $\mathrm{R}_{0}$. It weights the relative importance of the temperature contribution of each individual A-ANN to the overall ambient temperature, making each A-ANN an iterative consideration metric until a desired output is reached. The Scale of the experiment could be much larger in tune with the number of neurons that we see implemented, Figure 2 may expand in number without limit but also increasing the learning time required to exhaust a desired output. Each neuron is a threshold gate producing an activation value $\mathrm{V}_{\mathrm{k}}$. The larger the input values/synaptic weights the larger the activation value. $\mathrm{O}_{\mathrm{y}}$ Is actuator control decision and is calculated as follows:

$$
o_{y}=\left\{\begin{array}{c}
\text { Heat High if } V_{k} \geq \theta * 2 \\
\text { Heat Med if } V_{k} \geq \theta * 1.5 \\
\text { Heat Low if } V_{k} \geq \theta \\
\text { Heat Off if } V_{k}<\theta
\end{array}\right.
$$

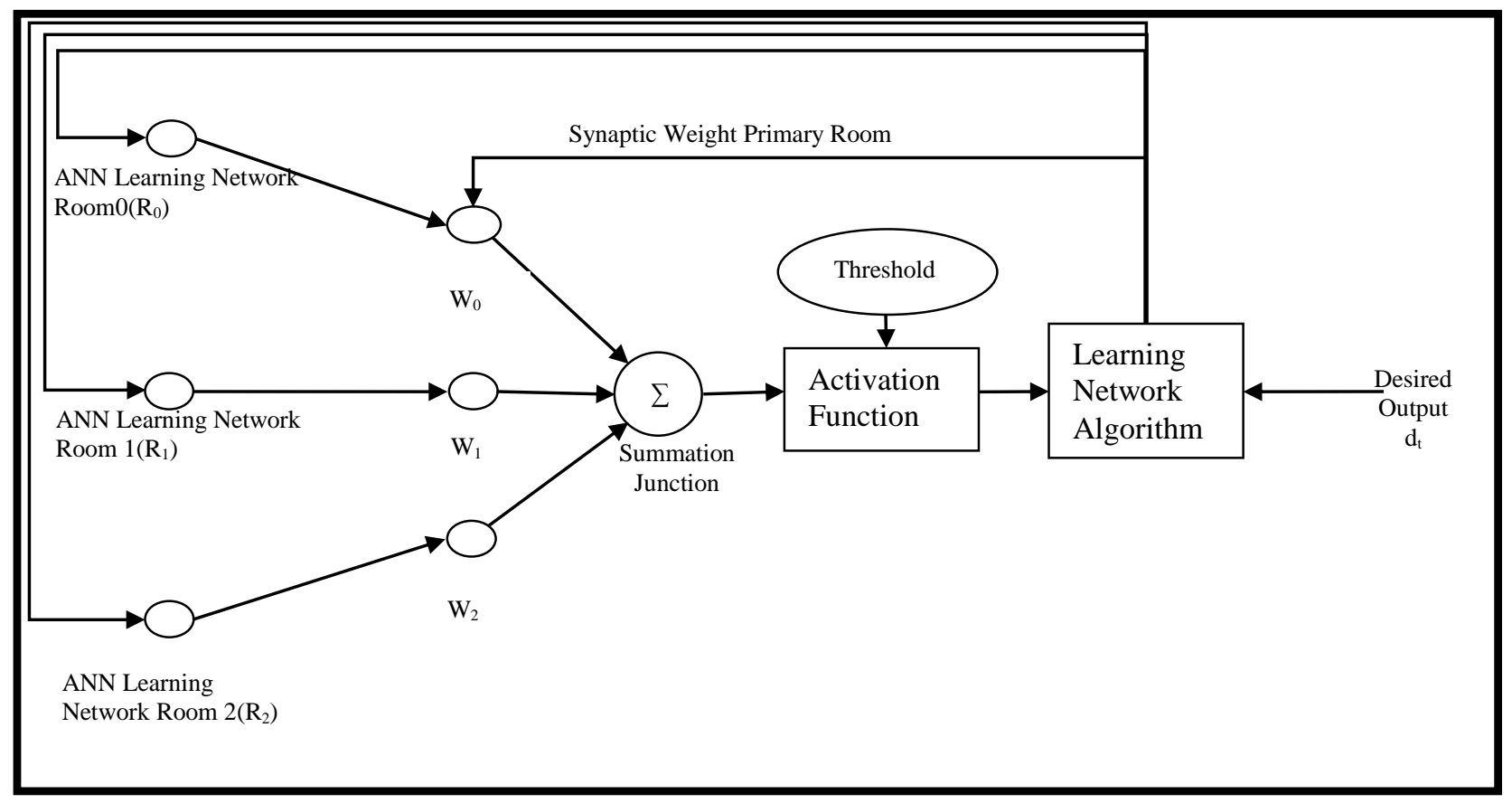

Figure 2. Synaptic Weight Adjustment 
We calculate the rate of change, $\mathrm{c}$, of a linear regression line for historic indoor temperature as follows:

$$
c=\frac{\sum\left(x-x^{\prime}\right)\left(y-y^{\prime}\right)}{\sum\left(x-x^{\prime}\right)^{2}}
$$

Using $\mathrm{c}$ we can determine the rate by which alterations to synaptic weights affect internal temperature. A positive $\mathrm{c}$ indicates that synaptic weight alterations have a beneficial effect on the delta in desired and actual temperature. A negative $\mathrm{c}$ indicates that synaptic weight alterations have a detrimental effect on the delta in desired and actual temperature. A large $\mathrm{c}$ (positive or negative) indicates numerous training cycles are required. A small $\mathrm{c}$ indicates that the selection of weights is close to optimal. In order to control the rate of learning we define a user configurable learning rate constant $r$. The selection of an appropriate learning rate is critical for the effective operation of the algorithm. If the learning rate is too low the network learns very slowly. If the learning rate is too high weights diverge, resulting in little learning. We define the error correction, $\Delta \mathrm{W}$, as the product of $\mathrm{c}$ and $\mathrm{r}$.

\section{SYSTEM EVALUATION}

Using the proposed neural network architecture, a scaled test model was created consisting of Arduino prototyping software andesp 8266 micro controllers with embedded Wi-Fi. JSON structured restful web programming interface calls provide a communication medium to push and pull information from the sensors. A relay was configured to actuate the heating and cooling system states. The intention is to recreate in scale for issues experienced as in (Gopika, 2015), (Vatanparvar, Burago, \& Abdullah, 2018), (Kim, 2018) and (Ardiyanto, et al, 2018). Figure 3 illustrates the configuration of the scaled model. Figure 3 illustrates the configuration of the scaled model which consists of 3 areas:

Meeting Room 1 - This is the main area for evaluation by the experiment. There is no temperature actuation (heating/cooling) in this room. The temperature of this room is dependent on the ambient temperature from both the Open Plan Area and Meeting Room 2. The room has a temperature sensor which provides readings to the C-ANN. Meeting Room 2 - The temperature actuation for configuration is controlled in this room. The room temperature is controlled by A-ANN policy which actuates both a heater and window which can be opened or closed. The room also has a temperature sensor which provides readings to the C-ANN. Open Plan Area- This area is the main working area. From an ambient temperature viewpoint, it connects the temperature actuation of Meeting Room 2 and Meeting Room 1. The area also has a temperature sensor which provides readings to the C-ANN.

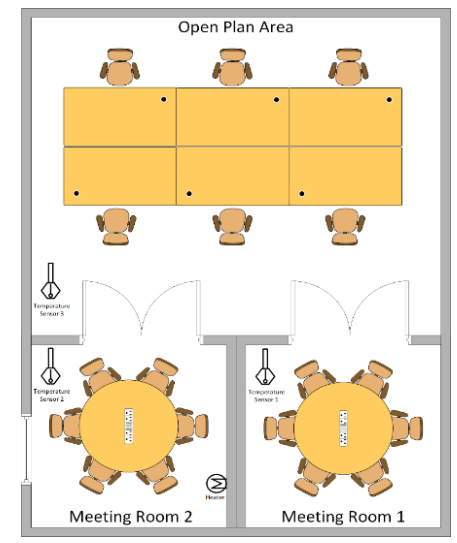

Figure 3. Scaled Office Configuration

In order to evaluate the operation of the approach 20 tests were undertaken. A sample representative test is described in detail to illustrate the operation of the approach. During the test the outdoor temperature ranged from a minimum of $17.06^{\circ}$ to a maximum of $18.27^{\circ}$. The desired ambient temperature for all room was a minimum of $20^{\circ}$ to a maximum of $27^{\circ}$. The A-ANN and C-ANN configurations are considered trained when the ambient temperature in all rooms was between the minimum and maximum values. The initial starting temperatures were Meeting Room $2=17.0^{\circ}$, Meeting Room $1=16.938^{\circ}$ and an external temperature of $17.125^{\circ}$. 
The experiment has 3 phases (1) Initial Analysis (2) ANN based temperature optimisation (3) ambient temperature dispersal. In the initial phase the C-ANN and A-ANN configurations use a coarse-grained mechanism to obtain optimal configuration. Actuators (heater/window opening) are configured using a preliminary rules-based policy to achieve a general optimal temperature. In this configuration the internal room temperatures of $17.0^{\circ}$ and $16.938^{\circ}$ are below optimal. The temperatures are also slightly below the external temperature of $17.125^{\circ}$. In the initial phase both actuators are activated; window is opened and heater is turned on. Figure 4 illustrates the external temperature, open plan area temperature and Meeting Room 1 temperature. The results illustrate that after 8 minutes the temperature of both internal areas has risen above the external temperature. At this point the window is automatically closed. The results also illustrate the increasing variation in temperature between the open plan area at $18.31^{\circ}$ and the Meeting Room 1 temperature of $17.188^{\circ}$.

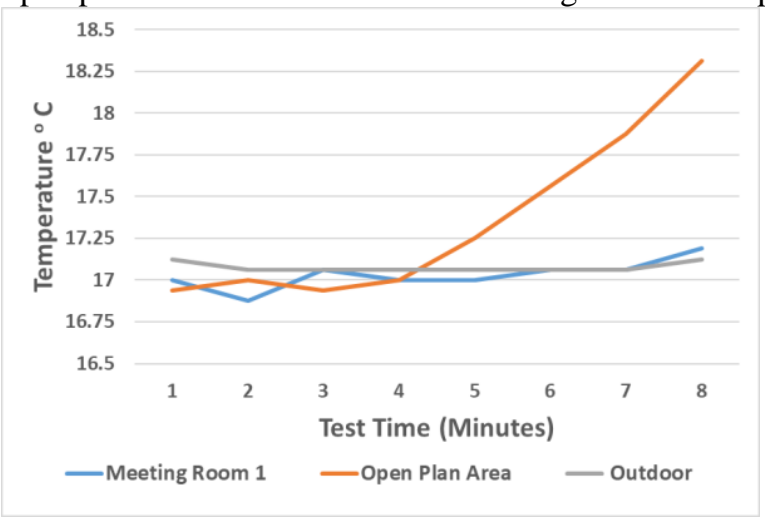

Figure 4. Test Phase 1 (0-8 Mins) Temperatures

In the second phase the external window has been closed and the C-ANN and A-ANN control the ambient internal temperature. The activation value controls the actuation of the heater. If the activation value is greater than 0 the heater is turned on. If the activation value is less than 0 the heater is turned off. Figure 5 illustrates the activation value during this test phase. Initially the activation value is 2.046 resulting in "Heater On". After 18 minutes the activation reaches its maximum value of 84.446 . The activation value remains above 0 until 40 minutes when it achieves the value -3.486. As this is a negative activation value the heater is turned off.

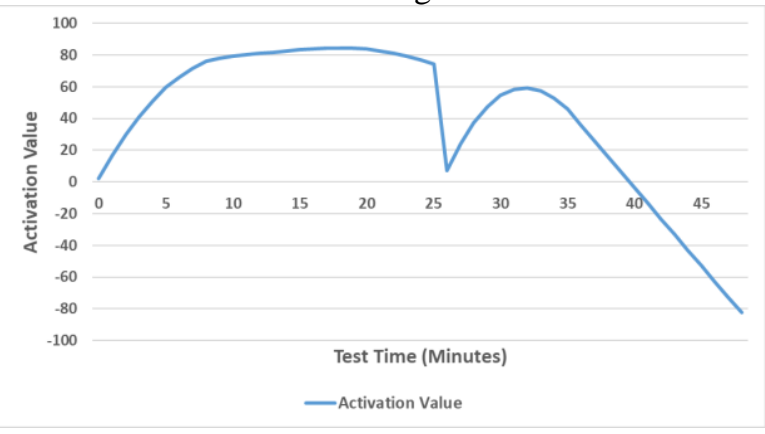

Figure 5. Test Phases 1 and 2 (0-50 Mins) Activation Value

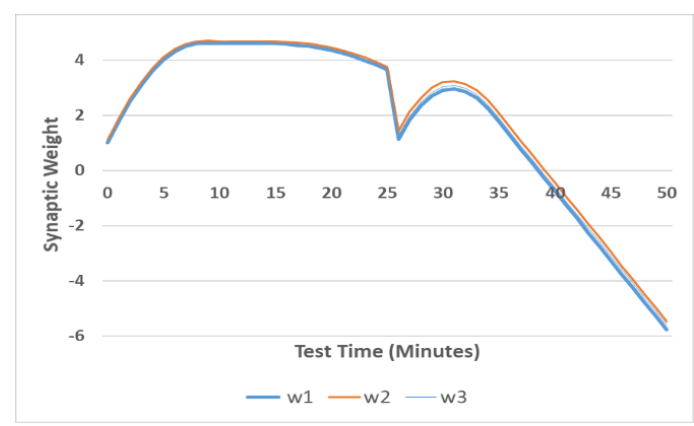

Figure 6. Test Phases 1 and 2 (0-50 Mins) synaptic Weights 
Starting with initial synaptic weights $\mathrm{w} 1=0.997$, w2 $=1.105$ and $\mathrm{w} 3=1.055$ the weights increase significantly in the first 0-8 second period. Increasing weights produce a more aggressive reaction from the $\mathrm{C}-\mathrm{ANN}$ and A-ANN meaning it is more likely the heater will be in state "On". The synaptic weights reach a maximum value of $\mathrm{w} 1=4.608, \mathrm{w} 2=4.692$ and $\mathrm{w} 3=4.647$ after 10 minutes, 9 minutes and 14 minutes respectively. There is a general decrease in all 3 synaptic weights until 25 minutes when there is a sudden reduction to $\mathrm{w} 1=1.143, \mathrm{w} 2=1.44$ and $\mathrm{w} 3=1.289$. In order to explain the sudden decrease in weight adjustment and resulting weights (Figure 6) we analyse the change in configuration temperature in Figure 7.

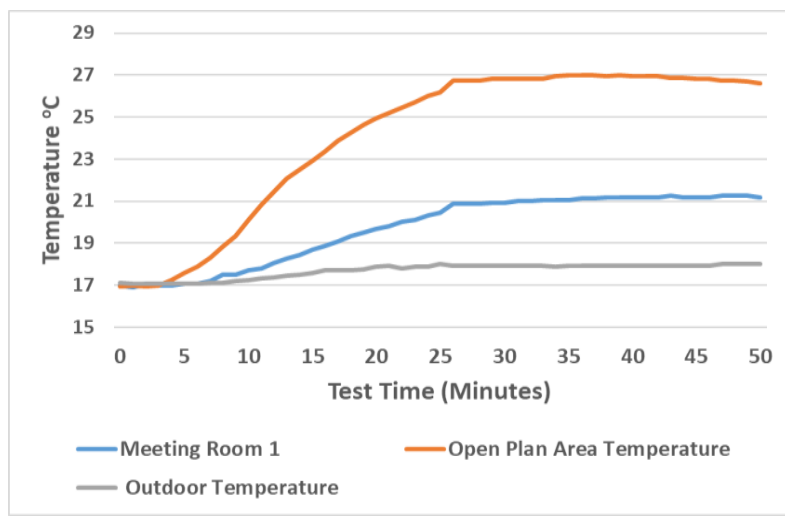

Figure 7. Test Phases 1 and 2 (0-50 Mins) Temperatures

Figure 7 illustrate that between 25 and 27 minutes there is a sudden increase in both the open plan area and meeting room 1 temperature. The open plan area room temperature increases from $20.3^{\circ}$ to $20.9^{\circ}$. In the same interval the temperature increases from $25.68^{\circ}$ to $26.8^{\circ}$. The C-ANN and A-ANN implementation react to the sudden increase in temperature by reducing the weight adjustment and thereby the weights. For the next 3 minutes the temperature of the open plan area and Meeting Room 1 remain constant at $20.9^{\circ}$ and $26.8^{\circ}$ respectively. As the temperature of Meeting Room 1 is below the required value the weigh increase is increased. After 40 minutes the activation value is reduced to a negative value and the heater is turned off. Figure 8 illustrates the external, open plan area and meeting room temperature for all the test phases.

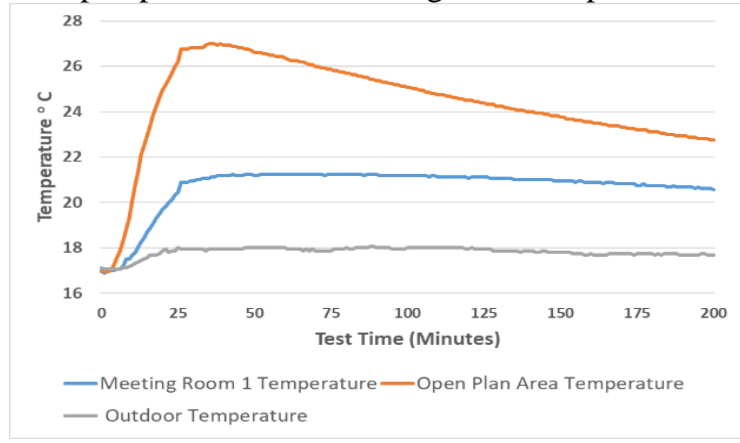

Figure 8. Complete Test Temperatures

In phase 1 (0-8 minutes) the window is open and the heater is on as the external temperature exceeds the internal temperature. After 8 minutes the window is closed as the internal temperature exceeds the external temperature. Between 9 minutes and 40 minutes the heater remains on as the activation value of the C-ANN and A-ANN configurations is greater than 0 . After 40 minutes the activation value is negative and the heater is turned off. The temperature of the open plan area reduces from a maximum of $26.938^{\circ} \mathrm{C}$ at 42 minutes to $22.75^{\circ} \mathrm{C}$ at the end of the test. The temperature of Meeting Room 1 reduces from a maximum of $21.25^{\circ} \mathrm{C}$ at 43 minutes to $20.563^{\circ} \mathrm{C}$ at the end of the test. 


\section{CONCLUSION}

This work proposes a federated ANN implementation which is influenced by environmental control factors to optimise ambient temperature within an office area. The configuration introduces two new architectural components an Agent ANN (A-ANN) and a Coordinating ANN (C-ANN). The A-ANN operates with a degree of autonomy with some federated influence from the C-ANN. The C-ANN considers the optimal ambient temperature of the entire open plan area. The work presents an experimentally based evaluation of the architecture using a physical scaled model. The model enables the analysis of dispersion of heat between rooms in an office environment consisting of meeting rooms and a central open plan area. The work illustrates how a federated ANN approach consisting of both centralised and distributed intelligence can learn the optimal of HVAC actuators so as to optimise thermal flow throughout the office space.

\section{REFERENCES}

Ardiyanto, D., Pipattanasomporn, M., Rahman, S., Hariyanto, N., \& Suwarno, N., 2018. Occupant-based HVAC Set Point Interventions for Energy Savings in Buildings. Green Energy for Sustainable Development. pp.1-6

Edenhofer and S. Kadner, S. K. Seyboth, A. Adler, I. Baum, S. Brunner, P. Eickemeier, B. Kriemann, J. Savolainen, S. Schlömer, C. von Stechow, T. Zwickel and J.C. Minx. 2014. Climate Change 2014: Mitigation of Climate Change. Cambridge University Press, Cambridge, United Kingdom and New York, NY, USA. pp. 671-739.

Gopika, S., 2015. Agent Based HVAC Optimization Model for Building Energy Efficiency. IEEE $9^{\text {th }}$ International Conference on Intelligent Systems and Control (ISCO). pp.1-5.

Hsiung, P., \&Lin, C., 2018. Cost optimization of electrical usage with customizable comfort considerations. IEEE International Conference on Applied System Invention (ICASI), Chiba. pp.172-175.

Kim, Y., 2018. Optimal Price Based Demand Response of HVAC Systems in Multizone Office Buildings Considering Thermal Preferences of Individual Occupants Buildings. IEEE Transactions on Industrial Informatics, vol. 14, no. 11, pp. 5060-5073.

Matta, K., Rehu, J., Tanner, H., Kansala, K., 2017. Building Intelligence - Home operating system for smart monitoring and control. IEEE Conference on Electro Information Technology (EIT). pp. 245-24.

Perry, R., Fallon, E., Fallon, S., \& Qiao., Y. 2017. A Hierarchical Learning System for Ambient Environmental Control of Open Plan Buildings. UKSim-AMSS 20th International Conference on Modelling \& Simulation. pp.21-27.

Priya Bhavesh, P. Karhade and Sulbha Rajkumar Hawa. 2016. Optimisation of HVAC system using programmable controllers and data acquisition. International conference on Internet of things and Applications (IOTA). pp.254-258.

Ueda, K., \& Shimonaka, S., 2017.Quantitative Evaluation of Intellectual Productivity Considering Depth of Concentration.IEEE International Conference on Systems, Man, and Cybernetics (SMC), Banff, Canada.pp. 758-763.

Vatanparvar, K., Burago, I., \& Abdullah, M., 2018. Extended Range Electric Vehicle with Driving Behaviour Estimation in Energy Management. IEEE Transaction on Smart Grid, vol. 14, no. 8. pp.2959 - 2968 